PROCEEDINGS OF THE

AMERICAN MATHEMATICAL SOCIETY

Volume 134, Number 5, Pages 1425-1434

S 0002-9939(05)08100-1

Article electronically published on October 13, 2005

\title{
A BOUND FOR RATIOS OF EIGENVALUES OF SCHRÖDINGER OPERATORS WITH SINGLE-WELL POTENTIALS
}

\author{
MIKLÓS HORVÁTH AND MÁRTON KISS \\ (Communicated by Carmen C. Chicone)
}

\begin{abstract}
For Schrödinger operators with nonnegative single-well potentials ratios of eigenvalues are extremal only in the case of zero potential. To prove this, we investigate some monotonicity properties of Prüfer-type variables.
\end{abstract}

\section{INTRODUCTION}

Consider the Schrödinger operator

$$
-y^{\prime \prime}+q(x) y=\lambda y
$$

on the interval $[0, \pi]$ with Dirichlet boundary conditions. If $q \in L_{1}(0, \pi)$ is realvalued, then the spectrum consists of a growing sequence of infinitely many points, $\lambda_{1}, \lambda_{2}, \ldots$; see for example in [3]. Moreover, if $q(x)$ is nonnegative, $\lambda_{n} \geq n^{2}$ (as it is seen later, for example, from (2.7) and Lemma 2.1).

Ashbaugh and Benguria in 2 proved the bound

$$
\frac{\lambda_{n}}{\lambda_{1}} \leq n^{2}
$$

for nonnegative potentials. They also examined the ratio of two arbitrary eigenvalues, and found

$$
\frac{\lambda_{n}}{\lambda_{m}} \leq\left\lceil\frac{n}{m}\right\rceil^{2}
$$

where $\lceil x\rceil$ denotes the smallest integer greater than or equal to $x$. To show that this estimate is optimal, they constructed multiple-well examples which came arbitrarily near to attain the bound. They formulated the conjecture that if the potential is nonnegative and convex, then

$$
\frac{\lambda_{n}}{\lambda_{m}} \leq \frac{n^{2}}{m^{2}}, \quad n \geq m,
$$

holds. In this paper we prove more. Namely, we only need that the potential $q \geq 0$ be single-well. This means that there is a point $a \in[0, \pi]$ such that $q$ is decreasing

Received by the editors December 5, 2003 and, in revised form, December 10, 2004 and December 14, 2004.

2000 Mathematics Subject Classification. Primary 34L15, 34B24.

Key words and phrases. Schrödinger operator, eigenvalues.

This research was supported by the Hungarian NSF Grants OTKA T 32374, T 37491 and T 47035 . 
in $[0, a]$ and increasing in $[a, \pi]$ (see in [1]). Our proof relies on some monotonicity properties of the Prüfer-type variables $\varphi$ and $r$ from (2.3)-(2.4).

\section{The Main STATEMEnt}

Denote by $y(x, z)$ the unique solution of the initial value problem

$$
\begin{gathered}
-y^{\prime \prime}+q(x) y=z^{2} y, \quad x \in[0, \pi], \quad z>0, \\
y(0)=0, y^{\prime}(0)=1,
\end{gathered}
$$

and let us introduce Prüfer-type variables:

$$
\begin{gathered}
y(x, z)=\frac{r(x, z)}{z} \sin \varphi(x, z), \\
y^{\prime}(x, z)=r(x, z) \cos \varphi(x, z), \\
\varphi(0, z)=0,
\end{gathered}
$$

where $r(x, z)>0$, and we denote by prime the derivative with respect to $x$ (and by dot the derivative with respect to $z$ ). Define further

$$
\psi=\frac{\varphi}{z} \text {. }
$$

An easy computation shows that for these variables the following equations hold:

$$
\begin{aligned}
\varphi^{\prime} & =z-\frac{q}{z} \sin ^{2} \varphi, \\
\frac{r^{\prime}}{r} & =\frac{q}{z} \sin \varphi \cos \varphi .
\end{aligned}
$$

Remark. These formulae hold in the usual sense at the continuity points of $q$ and in both half-sided senses at the jumps of $q: \varphi_{ \pm}^{\prime}(x, z)=z-\frac{q(x \pm 0)}{z} \sin ^{2} \varphi(x, z)$, and analogously for $r$.

It is obvious that $y=0$ iff $\sin \varphi=0$, hence $z^{2}$ is an eigenvalue iff $\varphi(\pi, z)$ is a multiple of $\pi$. Denote by $z_{n}$ the square root of $\lambda_{n}$.

Lemma 2.1. $\varphi\left(\pi, z_{n}\right)=n \pi$.

Proof. See equation (2.7) in Ashbaugh and Benguria [2].

Our idea is to show that (under certain conditions) $\psi(x, z)$ is a monotone increasing function in $z$, since this will imply (1.4).

Theorem 2.2. Let $q(x) \geq 0$ be monotone decreasing in $\left[0, x_{0}\right]$. Then $\dot{\psi}\left(x_{0}, z\right) \geq 0$, i.e., $\psi\left(x_{0}, z\right)$ is a monotone increasing function in $z>0$. If there is a $z>0$ with $\dot{\psi}\left(x_{0}, z\right)=0$, then $q=0$ in $\left(0, x_{0}\right]$.

This theorem implies various results for different boundary conditions. For example, we mention the following corollary.

Corollary 2.3. Consider equation (1.1) with the Dirichlet-Neumann boundary conditions

$$
y(0)=y^{\prime}(\pi)=0 .
$$


If the potential $q$ is nonnegative and decreasing, then for the $m$-th and $n$-th eigenvalues with $m \leq n$,

$$
\frac{\lambda_{n}}{\lambda_{m}} \leq \frac{(2 n-1)^{2}}{(2 m-1)^{2}}
$$

and if for two different $m$ and $n$ equality holds, then $q=0$ in $(0, \pi]$.

The proof will be given in Section 3 .

The main statement of this paper reads as follows:

Theorem 2.4. Consider equation (1.1) with the Dirichlet boundary conditions

$$
y(0)=y(\pi)=0 .
$$

If the potential $q$ is nonnegative and single-well, then for the $m$-th and $n$-th eigenvalues with $m \leq n$,

$$
\frac{\lambda_{n}}{\lambda_{m}} \leq \frac{n^{2}}{m^{2}}
$$

and if for two different $m$ and $n$ equality holds, then $q=0$ in $(0, \pi)$.

The proof will be given in Section 4 .

\section{The proof of Theorem 2.2}

Lemma 3.1. If $q(x)$ is monotone decreasing in $\left[0, x_{0}\right]$, then (for $\left.z>0\right) \varphi\left(x_{0}, z\right)$ is a strictly monotone increasing function of $x$ in $\left[0, x_{0}\right]$. Moreover, $\varphi_{ \pm}^{\prime}(x, z)>0$ for $z>0$.

Proof. Fix $z$. From (2.7) if $\varphi_{ \pm}^{\prime}(\tilde{x}, z) \leq 0$, then $q(x) \geq z^{2}$ for $x<\tilde{x}$. Through $y^{\prime \prime}=\left(q-z^{2}\right) y, y$ is convex, positive and increasing, so $y^{\prime}>0$, and by that, $\varphi(x, z)<\frac{\pi}{2}$ for $x<\tilde{x}$. For small $x>0, \varphi_{ \pm}^{\prime}(0, z)>0$ (see (2.7)). The function $\varphi_{ \pm}^{\prime}(x, z)$ is continuous at the continuity points of $q(x)$, and (since $q(x)$ is monotone decreasing) cannot jump downward. Thus if somewhere $\varphi_{ \pm}^{\prime}(x, z)$ is negative or zero, there exists a point $x_{2} \in(0, \tilde{x}]$ where $\varphi_{-}^{\prime}\left(x_{2}, z\right)=0$ and $\varphi_{ \pm}^{\prime}(x, z)>0$ for $x<x_{2}$. Choose an arbitrary point $x_{1} \in\left(0, x_{2}\right)$, then $0<\varphi(x, z)<\frac{\pi}{2}$ in $\left[x_{1}, x_{2}\right]$ and

$$
(z \cot \varphi(x, z))^{\prime}=q(x)-z^{2}-(z \cot \varphi(x, z))^{2}
$$

in $\left[x_{1}, x_{2}\right]$. Now we have

$$
(\cot \varphi)_{ \pm}^{\prime}(x, z)=\frac{-1}{\sin ^{2} \varphi} \varphi_{ \pm}^{\prime}(x, z),
$$

which implies $\sqrt{q(x)-z^{2}}<z \cot \varphi(x, z)$ for $x \in\left[x_{1}, x_{2}\right)$ and $\sqrt{q\left(x_{2}-0\right)-z^{2}}=$ $z \cot \varphi\left(x_{2}, z\right)$. We show that this is not possible. Indeed, choose $x_{3}$ arbitrarily from 
$\left[x_{1}, x_{2}\right)$. From (3.1) we get

$$
\begin{aligned}
& {\left[\log \left(z \cot \varphi(x, z)-\sqrt{q(x)-z^{2}}\right)\right]_{x_{1}}^{x_{3}} } \\
= & \int_{x_{1}}^{x_{3}} \frac{\mathrm{d}\left(z \cot \varphi(x, z)-\sqrt{q(x)-z^{2}}\right)}{z \cot \varphi(x, z)-\sqrt{q(x)-z^{2}}} \\
= & \int_{x_{1}}^{x_{3}} \frac{q(x)-z^{2}-(z \cot \varphi(x, z))^{2}}{z \cot \varphi(x, z)-\sqrt{q(x)-z^{2}}} \mathrm{~d} x \\
- & \int_{x_{1}}^{x_{3}} \frac{\mathrm{d} q(x)}{2 \sqrt{q(x)-z^{2}}\left(z \cot \varphi(x, z)-\sqrt{q(x)-z^{2}}\right)} \\
\geq & \int_{x_{1}}^{x_{3}}-\left(z \cot \varphi(x, z)+\sqrt{q(x)-z^{2}}\right) \mathrm{d} x
\end{aligned}
$$

by the monotonicity of $q$. Now $z \cot \varphi(x, z)$ is continuous and bounded in $\left[x_{1}, x_{2}\right)$, thus $-\left(z \cot \varphi(x, z)+\sqrt{q(x)-z^{2}}\right)$ is bounded from below, and hence

$$
\left[\log \left(z \cot \varphi(x, z)-\sqrt{q(x)-z^{2}}\right)\right]_{x_{1}}^{x_{3}} \geq K
$$

with $K$ independent of $x_{3}$. If we let $x_{3}$ approach $x_{2}$, this implies that

$$
z \cot \varphi\left(x_{2}, z\right)>\sqrt{q\left(x_{2}-0\right)-z^{2}},
$$

i.e., $\varphi_{-}^{\prime}\left(x_{2}, z\right)>0$, a contradiction.

In the following formulae we sometimes write $\varphi(x)$ instead of $\varphi(x, z)$.

\section{Lemma 3.2.}

$$
\dot{\varphi}(x)=\int_{0}^{x}\left(1+\frac{q(t)}{z^{2}} \sin ^{2} \varphi(t)\right) e^{-\int_{t}^{x} \frac{q}{z} \sin 2 \varphi} \mathrm{d} t .
$$

Proof. Differentiate equation (2.7) with respect to $z$ :

$$
\dot{\varphi}^{\prime}(x, z)=1+\frac{q(x)}{z^{2}} \sin ^{2} \varphi(x)-\frac{q(x)}{z} \sin 2 \varphi(x, z) \dot{\varphi}(x, z) .
$$

This is a linear differential equation in $x \rightarrow \dot{\varphi}(x, z)$. Multiplying both sides by $e^{\int_{0}^{x} \frac{q}{z} \sin 2 \varphi}$, we have

$$
\left(\dot{\varphi}(x, z) e^{\int_{0}^{x} \frac{q}{z} \sin 2 \varphi}\right)^{\prime}=\left(1+\frac{q(x)}{z^{2}} \sin ^{2} \varphi(x, z)\right) e^{\int_{0}^{x} \frac{q}{z} \sin 2 \varphi} .
$$

Using $\dot{\varphi}(0)=0$, we get (3.4).

Remark. From (2.8) we can rewrite (3.4):

$$
\dot{\varphi}(x)=\int_{0}^{x}\left(1+\frac{q(t)}{z^{2}} \sin ^{2} \varphi(t)\right) \frac{r^{2}(t)}{r^{2}(x)} \mathrm{d} t .
$$

From equation (3.7) it is obvious that $\varphi(x, z)$ is strictly monotone increasing in $z$. 


\section{Corollary 3.3.}

$$
\dot{\psi}(x)=\frac{2}{r^{2}(x) z^{2}} \int_{0}^{x} r^{2}\left(\frac{q}{z} \sin ^{2} \varphi-\frac{q}{z} \varphi \sin \varphi \cos \varphi\right) .
$$

Proof.

$$
\begin{aligned}
& \dot{\psi}(x)=\frac{\dot{\varphi}(x)}{z}-\frac{\varphi(x)}{z^{2}} \\
= & \frac{1}{r^{2}(x) z^{2}}\left\{\int_{0}^{x} r^{2}(t)\left[2\left(z-\varphi^{\prime}(t)\right)+\varphi^{\prime}(t)\right] \mathrm{d} t-r^{2}(x) \varphi(x)\right\} \\
= & \frac{2}{r^{2}(x) z^{2}} \int_{0}^{x}\left[r^{2}(t)\left(z-\varphi^{\prime}(t)\right)-r(t) r^{\prime}(t) \varphi(t)\right] \mathrm{d} t \\
= & \frac{2}{r^{2}(x) z^{2}} \int_{0}^{x} r^{2}\left(\frac{q}{z} \sin ^{2} \varphi-\frac{q}{z} \varphi \sin \varphi \cos \varphi\right) .
\end{aligned}
$$

From now on, we define the potential to be zero on $\left(x_{0}, \infty\right)$ and extend the definition of $\varphi, r$ and $\psi$ accordingly. Then $\varphi(x, z) \rightarrow \infty$ if $z$ is fixed and $x \rightarrow \infty$.

Lemma 3.4. If $0<|\varphi|<\frac{\pi}{2}$, then $\sin ^{2} \varphi-\varphi \sin \varphi \cos \varphi>0$.

Proof. This is a simple corollary of $\varphi<\tan \varphi$ if $0<\varphi<\frac{\pi}{2}$.

Corollary 3.5. Let $k \geq 0$ be an integer, $k \pi \leq c \leq k \pi+\frac{\pi}{2}, k \pi+\frac{\pi}{2} \leq d \leq(k+1) \pi$. Then for any fixed $z$

$$
\begin{gathered}
\int_{\varphi^{-1}(k \pi)}^{\varphi^{-1}(c)} r^{2}(t)\left(\frac{q}{z} \sin ^{2} \varphi-\frac{q}{z} \varphi \sin \varphi \cos \varphi\right) \geq-k \frac{\pi}{2}\left[r^{2}\right]_{\varphi^{-1}(k \pi)}^{\varphi^{-1}(c)}, \\
\int_{\varphi^{-1}\left(k \pi+\frac{\pi}{2}\right)}^{\varphi^{-1}(d)} r^{2}(t)\left(\frac{q}{z} \sin ^{2} \varphi-\frac{q}{z} \varphi \sin \varphi \cos \varphi\right) \geq-(k+1) \frac{\pi}{2}\left[r^{2}\right]_{\varphi^{-1}\left(k \pi+\frac{\pi}{2}\right)}^{\varphi^{-1}(d)},
\end{gathered}
$$

and equality holds iff $q=0$ in the corresponding open interval.

Proof.

$$
\begin{aligned}
& \int_{\varphi^{-1}(k \pi)}^{\varphi^{-1}(c)} r^{2}\left(\frac{q}{z} \sin ^{2} \varphi-\frac{q}{z} \varphi \sin \varphi \cos \varphi\right) \\
= & \int_{\varphi^{-1}(k \pi)}^{\varphi^{-1}(c)} r^{2}\left(\frac{q}{z} \sin ^{2}(\varphi-k \pi)-\frac{q}{z}(\varphi-k \pi) \sin \varphi \cos \varphi\right) \\
- & k \pi \int_{\varphi^{-1}(k \pi)}^{\varphi^{-1}(c)} r^{2}\left(\frac{q}{z} \sin \varphi \cos \varphi\right) .
\end{aligned}
$$


Now $(\varphi-k \pi)$ is between $-\frac{\pi}{2}$ and $\frac{\pi}{2}$, so, by the preceding lemma, the first term is positive, except when $q=0$. The second term is the same as in the right-hand side of (3.9) as we can easily see from (2.8).

The other part of the lemma can be proved in the same way:

$$
\begin{aligned}
& \int_{\varphi^{-1}\left(k \pi+\frac{\pi}{2}\right)}^{\varphi^{-1}(d)} r^{2}\left(\frac{q}{z} \sin ^{2} \varphi-\frac{q}{z} \varphi \sin \varphi \cos \varphi\right) \\
= & \int_{\varphi^{-1}\left(k \pi+\frac{\pi}{2}\right)}^{\varphi^{-1}(d)} r^{2}\left(\frac{q}{z} \sin ^{2}(\varphi-(k+1) \pi)-\frac{q}{z}(\varphi-(k+1) \pi) \sin \varphi \cos \varphi\right) \\
- & (k+1) \pi \int_{\varphi^{-1}\left(k \pi+\frac{\pi}{2}\right)}^{\varphi^{-1}(d)} r^{2}\left(\frac{q}{z} \sin \varphi \cos \varphi\right) \\
\geq & -(k+1) \pi \int_{\varphi^{-1}\left(k \pi+\frac{\pi}{2}\right)}^{\varphi^{-1}(d)} r r^{\prime}=-(k+1) \frac{\pi}{2}\left[r^{2}\right]_{\varphi^{-1}\left(k \pi+\frac{\pi}{2}\right)}^{\varphi^{-1}(d)}
\end{aligned}
$$

Corollary 3.6. Let $0 \leq C \leq \frac{\pi}{2}, 0 \leq D \leq \pi$. Then

$$
\begin{aligned}
& \int_{0}^{\varphi^{-1}(C)} r^{2}(t)\left(\frac{q}{z} \sin ^{2} \varphi-\frac{q}{z} \varphi \sin \varphi \cos \varphi\right) \geq 0, \\
& \int_{\varphi^{-1}\left(k \pi+\frac{\pi}{2}\right)}^{\varphi^{-1}\left(k \pi+\frac{\pi}{2}+D\right)} r^{2}(t)\left(\frac{q}{z} \sin ^{2} \varphi-\frac{q}{z} \varphi \sin \varphi \cos \varphi\right) \\
& \geq-(k+1) \frac{\pi}{2}\left[r^{2}\right]_{\varphi^{-1}\left(k \pi+\frac{\pi}{2}\right)}^{\varphi^{-1}\left(k \pi+\frac{\pi}{2}+D\right)},
\end{aligned}
$$

and equality holds iff $q=0$ in the corresponding open interval.

(3.11). follows from (3.9) with $k=0$. If $D \leq \frac{\pi}{2}$, then (3.12) is the same as (3.10). If not, it is the sum of (3.10) with $d=(k+1) \pi$ and (3.9) with $c=k \pi+\frac{\pi}{2}+D$ (and with $k$ replaced by $k+1$ ).

Lemma 3.7. $r\left(\varphi^{-1}\left(k \pi+3 \frac{\pi}{2}\right)\right) \leq r\left(\varphi^{-1}\left(k \pi+\frac{\pi}{2}\right)\right)$, if $k=0,1,2, \ldots$ Moreover, the function $r$ is monotone increasing betweeen $\varphi^{-1}(k \pi)$ and $\varphi^{-1}\left(k \pi+\frac{\pi}{2}\right)$ and is monotone decreasing between $\varphi^{-1}\left(k \pi+\frac{\pi}{2}\right)$ and $\varphi^{-1}((k+1) \pi)$.

Proof. Since the logarithmic function is strictly increasing, it is enough to prove

$$
\left[\log r^{2}\right]_{\varphi^{-1}\left(k \pi+\frac{\pi}{2}\right)}^{\varphi^{-1}\left(k \pi+\frac{3}{2} \pi\right)} \leq 0
$$


The monotonicity of $\log r^{2}$, (hence of $r$ ) follows from the sign of its derivative, $\frac{q}{z} \sin 2 \varphi$. By substituting $u=\varphi(x)$ :

$$
\begin{aligned}
& \int_{\varphi^{-1}\left(k \pi+\frac{\pi}{2}\right)}^{\varphi^{-1}((k+1) \pi)} \frac{2 r^{\prime}}{r}=\int_{\varphi^{-1}\left(k \pi+\frac{\pi}{2}\right)}^{\varphi^{-1}((k+1) \pi)} \frac{q}{z} \sin 2 \varphi \\
= & \int_{\varphi^{-1}\left(k \pi+\frac{\pi}{2}\right)}^{\left.\varphi^{-1}((k+1) \pi)\right)} \frac{q(x) \sin 2 \varphi(x)}{z^{2}-q(x) \sin ^{2} \varphi(x)} \varphi^{\prime}(x) \mathrm{d} x \\
= & \int_{k \pi+\frac{\pi}{2}}^{(k+1) \pi} \frac{q\left(\varphi^{-1}(u)\right) \sin 2 u}{z^{2}-q\left(\varphi^{-1}(u)\right) \sin ^{2} u} \mathrm{~d} u .
\end{aligned}
$$

Note that $\sin 2 u<0$ for $k \pi+\frac{\pi}{2}<u<(k+1) \pi$, while the denominator is always positive, as we have seen in Lemma 3.1. Hence if we replace $q$ by its minimum, $q\left(\varphi^{-1}((k+1) \pi)\right)$, the value of the fraction will increase:

$$
\begin{aligned}
\int_{\varphi^{-1}\left(k \pi+\frac{\pi}{2}\right)}^{\varphi^{-1}((k+1) \pi)} \frac{2 r^{\prime}}{r} & \leq \int_{k \pi+\frac{\pi}{2}}^{(k+1) \pi} \frac{q\left(\varphi^{-1}((k+1) \pi)\right) \sin 2 u}{z^{2}-q\left(\varphi^{-1}((k+1) \pi)\right) \sin ^{2} u} \mathrm{~d} u \\
& =\left[-\ln \left(z-\frac{q\left(\varphi^{-1}((k+1) \pi)\right)}{z} \sin ^{2} u\right)\right]_{k \pi+\frac{\pi}{2}}^{(k+1) \pi}
\end{aligned}
$$

The other part of the integral can be handled in an analogous way except that this time $\sin 2 u>0$ on the interval in question and we replace $q$ by its maximum:

$$
\int_{\varphi^{-1}((k+1) \pi)}^{\varphi^{-1}\left(k \pi+3 \frac{\pi}{2}\right)} \frac{2 r^{\prime}}{r} \leq\left[-\ln \left(z-\frac{q\left(\varphi^{-1}((k+1) \pi)\right)}{z} \sin ^{2} u\right)\right]_{(k+1) \pi}^{k \pi+3 \frac{\pi}{2}} .
$$

Summing up,

$$
\int_{\varphi^{-1}\left(k \pi+\frac{\pi}{2}\right)}^{\varphi^{-1}\left(k \pi+3 \frac{\pi}{2}\right)} \frac{2 r^{\prime}}{r} \leq\left[-\ln \left(z-\frac{q\left(\varphi^{-1}((k+1) \pi)\right)}{z} \sin ^{2} u\right)\right]_{k \pi+\frac{\pi}{2}}^{k \pi+3 \frac{\pi}{2}}=0
$$


Proof of Theorem 2.2. If $\varphi\left(x_{0}, z\right) \leq \frac{\pi}{2}$, then the statement of the theorem immediately follows from (3.8) and (3.11). If not, let $\varphi\left(x_{0}, z\right)=\frac{\pi}{2}+k \pi+D$, with $0 \leq D \leq \pi$ :

$$
\begin{aligned}
& \int_{0}^{\varphi^{-1}\left(\frac{\pi}{2}+k \pi+D\right)} r^{2}(t)\left(\frac{q}{z} \sin ^{2} \varphi-\frac{q}{z} \varphi \sin \varphi \cos \varphi\right) \mathrm{d} t \\
= & \int_{0}^{\varphi^{-1}\left(\frac{\pi}{2}\right)} r^{2}(t)\left(\frac{q}{z} \sin ^{2} \varphi-\frac{q}{z} \varphi \sin \varphi \cos \varphi\right) \mathrm{d} t \\
+ & \sum_{i=1}^{k} \int_{\varphi^{-1}\left(i \pi-\frac{\pi}{2}\right)}^{\varphi^{-1}\left(i \pi+\frac{\pi}{2}\right)} r^{2}(t)\left(\frac{q}{z} \sin ^{2} \varphi-\frac{q}{z} \varphi \sin \varphi \cos \varphi\right) \mathrm{d} t \\
+ & \int_{\varphi^{-1}\left(k \pi+\frac{\pi}{2}\right)}^{\varphi^{-1}\left(k \pi+\frac{\pi}{2}+D\right)} r^{2}(t)\left(\frac{q}{z} \sin ^{2} \varphi-\frac{q}{z} \varphi \sin \varphi \cos \varphi\right) \mathrm{d} t .
\end{aligned}
$$

Here every term is nonnegative by Corollary 3.6 and Lemma 3.7 and if their sum is zero, then $q$ has to be zero in the whole interval $\left(0, x_{0}\right]$.

Proof of Corollary 2.3. By the current boundary conditions,

$$
z_{n} \psi\left(\pi, z_{n}\right)=\left(n-\frac{1}{2}\right) \pi .
$$

Let $m$ be less than $n$. Then $\frac{(2 m-1) \pi}{2 z_{m}}=\psi\left(\pi, z_{m}\right) \leq \psi\left(\pi, z_{n}\right)=\frac{(2 n-1) \pi}{2 z_{n}}$, and thus $\frac{z_{n}}{z_{m}} \leq \frac{2 n-1}{2 m-1}$ and $\frac{\lambda_{n}}{\lambda_{m}} \leq \frac{(2 n-1)^{2}}{(2 m-1)^{2}}$. If equality holds, then $\psi\left(\pi, z_{m}\right)=\psi\left(\pi, z_{n}\right)$, and by Theorem 2.2 this implies that $q=0$ in $(0, \pi]$.

\section{The Proof of Theorem 2.4}

Let the potential $q(x)$ be monotone decreasing in $[0, a]$ and monotone increasing in $[a, \pi]$. Denote by $\tilde{q}(x)$ the reverse of the potential, i.e., $\tilde{q}(x)=q(\pi-x)$. Then $y\left(\pi-x, z_{n}\right)$ is an eigenfunction for the potential $\tilde{q}(x)$. Moreover, define

$$
\begin{gathered}
\tilde{y}\left(x, z_{n}\right)=(-1)^{n+1} \frac{y\left(\pi-x, z_{n}\right)}{r\left(\pi, z_{n}\right)}, \\
\tilde{r}\left(x, z_{n}\right)=\frac{r\left(\pi-x, z_{n}\right)}{r\left(\pi, z_{n}\right)},
\end{gathered}
$$

and

$$
\tilde{\varphi}\left(x, z_{n}\right)=n \pi-\varphi\left(\pi-x, z_{n}\right) .
$$

Then

$$
\begin{gathered}
\tilde{y}\left(0, z_{n}\right)=0, \\
\tilde{y}^{\prime}\left(0, z_{n}\right)=1,
\end{gathered}
$$

which means that $\tilde{y}\left(x, z_{n}\right)$ is the solution of the initial value problem (2.1)-(2.2) with $\tilde{q}$ instead of $q$. It is also simple that

$$
\tilde{y}\left(x, z_{n}\right)=\frac{\tilde{r}\left(x, z_{n}\right)}{z_{n}} \sin \tilde{\varphi}\left(x, z_{n}\right),
$$




$$
\begin{gathered}
\tilde{y}^{\prime}\left(x, z_{n}\right)=\tilde{r}\left(x, z_{n}\right) \cos \tilde{\varphi}\left(x, z_{n}\right), \\
\tilde{\varphi}\left(0, z_{n}\right)=0,
\end{gathered}
$$

which prove that $\tilde{r}$ and $\tilde{\varphi}$ are the Prüfer-variables for $\tilde{y}$. According to (2.6), let $\tilde{\psi}=\frac{\tilde{\varphi}}{z}$.

Proof of Theorem 2.4. Consider the function $\Psi(z)=\psi(a, z)+\tilde{\psi}(\pi-a, z)$. This is, by Theorem 2.2 the sum of two monotone increasing functions. By (4.3),

$$
z_{n} \Psi\left(z_{n}\right)=n \pi .
$$

Let $m$ be less than $n$. Then $\frac{m \pi}{z_{m}}=\Psi\left(z_{m}\right) \leq \Psi\left(z_{n}\right)=\frac{n \pi}{z_{n}}$, and thus $\frac{z_{n}}{z_{m}} \leq \frac{n}{m}$ and $\frac{\lambda_{n}}{\lambda_{m}} \leq \frac{n^{2}}{m^{2}}$. If equality holds, then $\Psi\left(z_{m}\right)=\Psi\left(z_{n}\right)$, hence $\psi\left(a, z_{m}\right)=\psi\left(a, z_{n}\right)$ and $\tilde{\psi}\left(\pi-a, z_{m}\right)=\tilde{\psi}\left(\pi-a, z_{n}\right)$. By Theorem 2.2 this implies that $q=0$ in $(0, a]$ and $\tilde{q}=0$ in $(0, \pi-a]$, i.e., $q=0$ in $(0, \pi)$.

\section{REMARKS}

Remark 1. If the potential is not monotone decreasing, then $\psi$ might not increase in $z$ at some point. For example, let $q$ be zero in $\left[0, \frac{2}{3} \pi\right]$ and 1 otherwise. Pick $z=3 / 2$. We can easily see that

$$
\begin{aligned}
& y\left(x, \frac{3}{2}\right)=\left\{\begin{array}{cc}
\frac{2}{3} \sin \frac{3}{2} x & \text { if } x \leq \frac{2}{3} \pi, \\
-\frac{2}{\sqrt{5}} \sin \frac{\sqrt{5}}{2}\left(x-\frac{2}{3} \pi\right) & \text { otherwise }
\end{array}\right. \\
& y^{\prime}\left(x, \frac{3}{2}\right)=\left\{\begin{array}{cc}
\cos \frac{3}{2} x & \text { if } x \leq \frac{2}{3} \pi \\
-\cos \frac{\sqrt{5}}{2}\left(x-\frac{2}{3} \pi\right) & \text { otherwise }
\end{array}\right.
\end{aligned}
$$

From (2.7)

$$
\varphi^{\prime}(x) \geq \frac{3}{2}-\frac{2}{3}>0
$$

It can be easily checked that $\varphi\left(\frac{2}{3} \pi, \frac{3}{2}\right)=\pi, \varphi\left(\pi, \frac{3}{2}\right) \approx 4.27083<\frac{3}{2} \pi$. Combining this with (2.8), we get

$$
r(x)=\exp \left(\int_{0}^{x} \frac{q}{z} \sin \varphi \cos \varphi\right)=\left\{\begin{array}{cl}
1 & \text { if } x \leq \frac{2}{3} \pi \\
\exp \left(\int_{\pi}^{\varphi(x)} \frac{\frac{2}{3} \sin v \cos v}{\frac{3}{2}-\frac{2}{3} \sin ^{2} v} \mathrm{~d} v\right) & \text { otherwise }
\end{array}\right.
$$

hence

$$
\begin{aligned}
\dot{\psi}(\pi) & =\frac{2}{r^{2}(\pi) z^{2}} \int_{0}^{\pi} r^{2}\left(\frac{q}{z} \sin ^{2} \varphi-\frac{q}{z} \varphi \sin \varphi \cos \varphi\right) \\
& =\frac{2}{r^{2}(\pi) z^{2}} \int_{\frac{2}{3} \pi}^{\pi} \frac{r^{2}\left(\frac{2}{3} \sin ^{2} \varphi-\frac{2}{3} \varphi \sin \varphi \cos \varphi\right)}{\frac{3}{2}-\frac{2}{3} \sin ^{2} \varphi} \varphi^{\prime},
\end{aligned}
$$

and substituting $\varphi(x)=u$ to this integral, we can numerically compute it (using Maple or Mathematica):

$$
\int_{\pi}^{4.27083} \exp \left(\int_{\pi}^{u} \frac{\frac{2}{3} \sin 2 v}{\frac{3}{2}-\frac{2}{3} \sin ^{2} v} \mathrm{~d} v\right) \frac{\left(\frac{2}{3} \sin ^{2} u-\frac{2}{3} u \sin u \cos u\right)}{\frac{3}{2}-\frac{2}{3} \sin ^{2} u} \mathrm{~d} u \approx-0.811
$$

which means that $\psi$ is not growing at $z=\frac{3}{2}$. 
Remark 2. We could ask whether the sum of the $\psi(\pi)$ 's belonging to the potential $q(x)$ and $q(\pi-x)$ is a monotone increasing function in $z$. But the last example shows that this is not necessarily the case either. Indeed,

$$
\dot{\psi}(\pi) \approx \frac{2}{r^{2}(\pi) z^{2}}(-0.811) \approx-0.292 .
$$

In a similar manner we can compute $\dot{\psi}\left(x, \frac{3}{2}\right)$ belonging to $q(\pi-x)$, which approximately equals 0.0306 , so the sum is also negative.

\section{REFERENCES}

[1] M. Ashbaugh and R. Benguria, Optimal lower bound for the gap between the first two eigenvalues of one-dimensional Schrödinger operators with symmetric single-well potentials, Proc. Amer. Math. Soc. (1989), 105 419-424. MR0942630 (89f:81028)

[2] M. Ashbaugh and R. Benguria, Optimal bounds for ratios of eigenvalues of one-dimensional Schrödinger operators with Dirichlet boundary conditions and positive potentials, Commun. Math. Phys (1989), 124 403-415. MR1012632(91c:34114)

[3] B. M. Levitan and I. S. Sargsjan, Sturm-Liouville and Dirac operators (in Russian), Nauka, Moscow, (1988). MR0958344 (90c:34028)

Department of Mathematical Analysis, Institute of Mathematics, Budapest UniverSity of Technology and Economics, H 1111 Budapest, Múegyetem RKp. 3-9, Hungary

E-mail address: horvath@math.bme.hu

Department of Mathematical Analysis, Institute of Mathematics, Budapest UniverSity of Technology and Economics, H 1111 Budapest, Múegyetem RKp. 3-9, Hungary

E-mail address: mkiss@math.bme.hu 